\title{
X-ray analysis of the short-range order in the ordered-alloy domains of epitaxial (Ga,In)P layers by diffraction anomalous fine structure of superlattice reflections
}

\author{
Dirk C. Meyer, Kurt Richter, and Peter Paufler \\ Institut für Kristallographie und Festkörperphysik, Fachrichtung Physik, Technische Universität Dresden, D-01062 Dresden, Germany \\ Gerald Wagner \\ Fakultät für Chemie und Mineralogie, Universität Leipzig, Linnéstrasse 3, D-04103 Leipzig, Germany
}

(Received 6 January 1999; revised manuscript received 19 February 1999)

\begin{abstract}
Ordered-alloy domains of epitaxially grown (Ga,In)P layers have been observed elsewhere using transmission electron microscopy and transmission electron diffraction. We used diffraction anomalous fine-structure (DAFS) experiments at superlattice reflections occurring in several $\langle 111\rangle$ directions to explore the short-range order around $\mathrm{Ga}$ atoms in such ordered domains in epitaxial (Ga,In)P layers grown on (001) GaAs substrates. The requirements for a reliable measurement of the reflection intensity depending on the photon energy are described. A quantitative DAFS analysis resulting in short-range order parameters is explained in detail. The local structure around $\mathrm{Ga}$ in the whole $(\mathrm{Ga}$,In)P layer $(F \overline{4} 3 \mathrm{~m})$ can be understood by a local structure model, while contrary to that the local structure around $\mathrm{Ga}$ atoms in the ordered regions $(R 3 \mathrm{~m})$ can be described by the values expected on the basis of the virtual-crystal model. [S0163-1829(99)09023-2]
\end{abstract}

\section{INTRODUCTION}

In epitaxial layers of III-V ternaries dependent on the process parameters during growth, small superlattice domains often come into being which are supposed to influence the electronic properties (e.g., gap energy) of this material and hence of the devices made of it. ${ }^{1}$ Therefore, the structure of the ordered domains has been studied by transmission electron microscopy (TEM) and transmission electron diffraction, ${ }^{2-4}$ the latter giving information about the size of the ordered domains as well. Mostly a CuPt-like structure of the column III elements sublattice is reported. This emphasis on one direction leads to a reduction from the original $F \overline{4} 3 \mathrm{~m}$ symmetry to a rhombohedral $R 3 \mathrm{~m}$ symmetry of the whole structure. Our diffraction anomalous fine-structure (DAFS) measurements are exclusively aimed at a quantitative characterization of the short-range order of the ordered domains. Therefore we used superlattice reflections, ${ }^{5,6}$ where only the relevant anomalous scatterers contribute to the DAFS. This is important because merely a small fraction (few percent) of the whole layer volume is subdued to the ordering, resulting in a negligibly small contribution to the DAFS of the main reflections.

In the present paper we discuss the experimental requirements of DAFS measurements at superlattice reflections, and of the DAFS analysis in detail referring to Arcon et al. ${ }^{7}$ Stragier et al., ${ }^{8}$ and Sorensen et al.,${ }^{9}$ who described the theory, experimental methods, analytical techniques and various applications of DAFS. Experiments for study of strained III-V epitaxial semiconductors by means of DAFS were reported by Proietti et al. ${ }^{10}$ Methodical aspects of a quantitative analysis of DAFS experiments with noncentrosymmetric single crystals were already reported in detail in Ref. 11.

\section{SPECIMENS}

The epitaxial $(\mathrm{Ga}, \mathrm{In}) \mathrm{P}$ layers were prepared by metalorganic-vapor-phase epitaxy as described elsewhere. ${ }^{12}$ The
(001) GaAs substrates were misoriented $2^{\circ}$ toward the azimuthal [010] direction. The samples investigated differed in growth temperatures, as can be seen from Table I. Ordering was found by TEM for all specimens but at different extent in [ $1 \overline{1} 1]$ and [ $\overline{1} 11]$ directions. Due to the shape of the observed superlattice reflections, the dimensions of the ordered domains were found to be wide spread in the range of a few micrometers. The ordered domains are mostly arranged like flat discs. In another case Morita et al. ${ }^{4}$ reported on lateral dimensions of the ordered regions in the range up to $10^{4} \mathrm{~nm}^{2}$. The percentage of the ordered volume was determined by quantitative x-ray phase analysis. For this purpose the superlattice reflection intensities were compared to those of the corresponding second order reflection intensities of (Ga,In)P $\{111\}$ lattice planes. Therefore intensities were calculated taking into consideration the appropriate corrections of x-ray diffractometry (absorption correction, polarization, and Lorentzian factors). It is advantageous that the secondorder reflections of the GaAs $\{111\}$ lattice planes are forbidden in case of nonanomalous scattering conditions. The results are given in Table II.

\section{EXPERIMENT}

DAFS experiments were carried out at the Hamburg Synchrotron Radiation Laboratory HASYLAB at the undulator beamline BW1 using a Si 111 double-crystal monochromator with fixed exit. The eight-circle diffractometer available at

TABLE I. Growth parameters of investigated specimens.

\begin{tabular}{ccc}
\hline \hline Specimen & Subtrate temperature & $(\mathrm{Ga}, \mathrm{In}) \mathrm{P}$ layer thickness \\
\hline $60 / 2$ & $650{ }^{\circ} \mathrm{C}$ & about $1.90 \mu \mathrm{m}$ \\
$69 / 2$ & $680^{\circ} \mathrm{C}$ & $1.69 \mu \mathrm{m}$ \\
$48 / 2$ & $720^{\circ} \mathrm{C}$ & $1.90 \mu \mathrm{m}$ \\
\hline \hline
\end{tabular}


TABLE II. Proportion of ordered volume (in vol \%) for two orientations of the $c$ axis.

\begin{tabular}{ccc}
\hline \hline & $\begin{array}{c}R 3 m c \text { axis } \\
\text { (hex. axis) parallel } \\
\text { Specimen }\end{array}$ & $\begin{array}{c}R 3 m c \text { axis } \\
\text { (hex. axis) parallel } \\
\text { 4 } 3 m[1 \overline{1} 1]\end{array}$ \\
\hline $60 / 2$ & $2.6 \pm 0.2$ & $0.3 \pm 0.2$ \\
$69 / 2$ & $8.7 \pm 0.2$ & $1.1 \pm 0.2$ \\
$48 / 2$ & $6.7 \pm 0.2$ & $1.2 \pm 0.2$ \\
\hline \hline
\end{tabular}

this measuring station was used to obtain the normal of the $\langle 111\rangle$ directions of the $(\mathrm{Ga}, \mathrm{In}) \mathrm{P}$ layers into the scattering plane (sample angle $\omega$, tilt angle of the sample goniometer $\psi)$. For tracking the Bragg angle $\theta$ versus energy $E$ in the range of $800 \mathrm{eV}$ above the $\mathrm{Ga} K$ absorption edge (about $10369 \mathrm{eV}$ ) to get the reflection intensity $I(E)$ the accuracy of the sample goniometer was sufficiently high $\left(0.001^{\circ}\right)$ because the superlattice reflections of $(\mathrm{Ga}, \mathrm{In}) \mathrm{P}$ were broad (a full width at half maximum of about $0.4^{\circ}$ in $\theta$ ). The reflection intensities and the Ga $K$ fluorescence intensities were recorded simultaneously by two thermoelectrically cooled Si-pin photodiodes ${ }^{13}$ using Keithley 427 and 428 current amplifiers for the photocurrent measurement, respectively. The Ga $K$ fluorescence intensity was used for the absorption correction. The incoming monochromatic beam was limited by slits $(2.62 \mathrm{~mm}$ in the $\omega$ direction, and $0.7 \mathrm{~mm}$ parallel to the $\omega$ axis) and monitored by an ionization chamber situated between the slits and the specimen. A 1.09-mm slit in front of the reflection detector (in the $\theta$ direction) reduced the fluorescence background. The energy-dependent background was measured near the Bragg angle of the superlattice reflection, and subtracted from the measured reflection intensity. The polarization vector of the incident radiation was parallel to the normal of the scattering plane. For the Bragg angle tracking of the specimen the sample goniometer was moved using a second-order polynomial $\omega(E) / \theta(E)$ which was obtained by a diffraction preexperiment to find out the peak positions at several energies in the scanning range. The DAFS experiments at the superlattice reflections ran within the energy range of 10200-11000 eV with energy steps of 2 $\mathrm{eV}$, and measuring times of 5-15 s depending on the reflection intensities. At a mean Bragg angle $\omega$ of $5^{\circ}$, about $60 \%$ of the radiation intensity interacts with the layer. For specimen 48/2, Fig. 1 shows the measured reflection intensities $I_{M}(E)$ of the superlattice reflections in [1 $\left.1 \overline{1} 1\right]$ and [111] directions. The simultaneously measured normalized $\mathrm{Ga} K$ fluorescence intensity of the (Ga,In)P layer is shown in Fig. 2. The Ga $K$ fluorescence from the GaAs substrate is negligible because of the maximum exit angle of only $3^{\circ}$ (there is about a $0.04-\mathrm{mm}$ path length in the layer) given by the position of the fluorescence detector.

\section{QUANTITATIVE DAFS ANALYSIS}

The quantitative DAFS analysis started with an absorption correction of the measured reflection intensities $I_{M}(E)$ because these intensities are superimposed by the $\mathrm{x}$-rayabsorption fine structure (XAFS) averaged over all anomalous scatterers of the specimen volume, which in our case

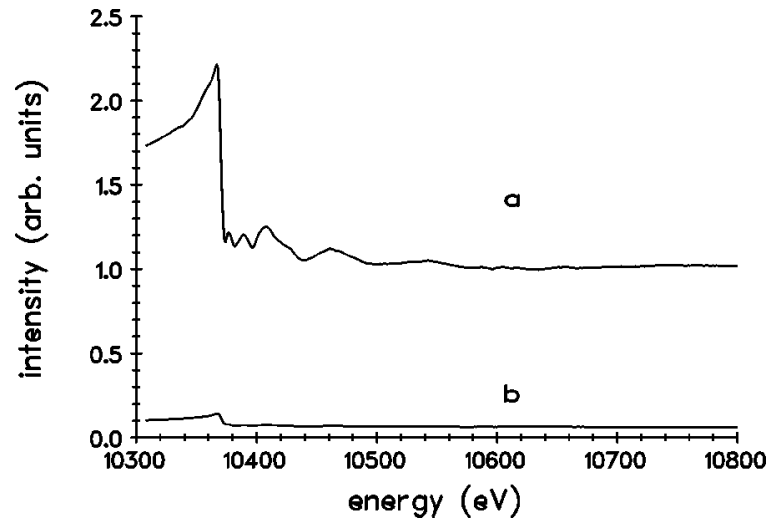

FIG. 1. Measured DAFS reflection intensities of the $R 3 m 003$ superlattice reflections vs photon energy, hexagonal $c$ axis parallel to [1 $1 \overline{1} 1]$ (a) and [ $\overline{1} 11]$ (b) directions of the sphalerite $F \overline{4} 3 m$ structure of specimen $48 / 2$.

were far more than the few atoms contributing to the superlattice reflections. The next steps were the calculation of the smoothed curve (without the fine structure) of reflected intensities and the adaptation of the medium run of the corrected measured intensity to gain the oscillating part of $I_{M}(E)$, from which the complex-valued fine structure function was obtained by applying an iterative Kramers-Kronig algorithm. Finally the short-range order parameters were calculated by modeling the theoretical fine-structure function and by comparing it with the experimental one as extracted from DAFS signals. The absorption correction was done by dividing the measured reflection intensities by an absorption correction factor $A(E)$ :

$$
I(E)=\frac{I_{M}(E)}{A(E)} .
$$

$A(E)$ can be calculated by integrating over the different possible path lengths of the x-ray photons in the layer of thickness $t$ :

$$
A(E)=\frac{1-\exp \left(\frac{-2 \mu(E) t}{\sin \theta \cos \psi}\right)}{\frac{2 \mu(E)}{\sin \theta \cos \psi}}
$$

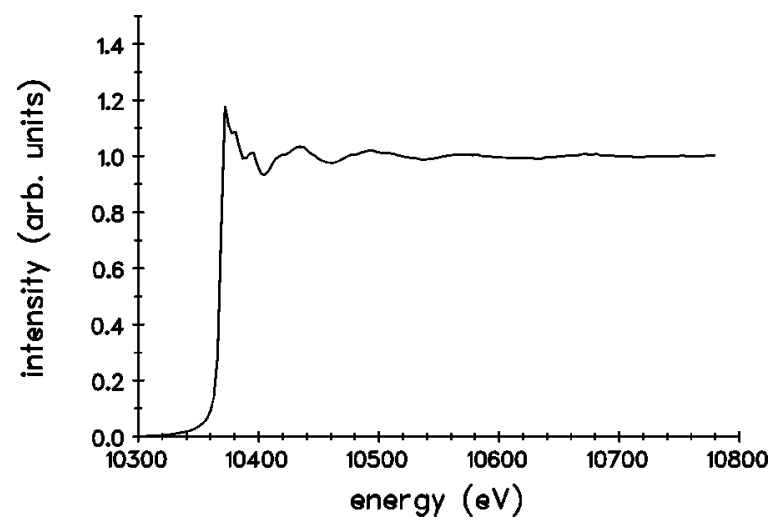

FIG. 2. Ga $K$ fluorescence intensity vs photon energy (background corrected and normalized) measured simultaneously with DAFS shown in Fig. 2. 


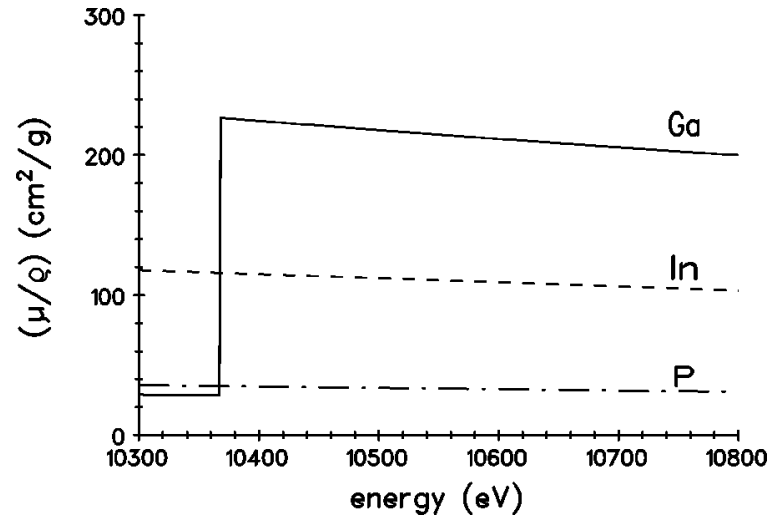

FIG. 3. Total mass absorption coefficients of the layer components versus photon energy (Ref. 14).

with

$$
\mu(E)=\mu_{e}(E)[1-\chi(E)]+\mu_{b}(E),
$$

where $\mu_{e}(\mathrm{E})$ is the absorption coefficient of the edge atom, $\mu_{b}(E)$ is the total absorption coefficient of all nonedge atoms, $\chi(E)$ is the XAFS contribution to the absorption coefficient of the edge atom, $\theta$ is the Bragg angle, and $\psi$ the tilting angle of the sample. The total absorption coefficient $\mu(E)$ of the (Ga,In)P layer was calculated using Eq. (3), including the mass absorption coefficients for the elements $\mathrm{P}$, $\mathrm{Ga}$, and In (Fig. 3), ${ }^{14}$ and a mass density $\rho=4.459 \mathrm{~g} / \mathrm{cm}^{3}$ according to Ref. 15. Inserting $\mu(E)$ into Eq. (2) then gives $A(E)$, as shown in Fig. 4. The corrected reflection intensities $I_{M}(E) / A(E)$ for the superlattice reflections of specimen $48 / 2$ are shown in Fig. 5. It was assumed that the ordered domains were equally distributed with depth. In fact a small change of thickness should influence the DAFS analysis only weakly. The reflection intensity $I(E)$ is proportional to the square of the structure factor, $|F(E)|^{2}$. The kinematic scattering approximation should be justified because of the small size of superlattice domains. For a description of superlattice reflections the structure factor had to be evaluated for the rhombohedral phase $R 3 \mathrm{~m}$ (space group number 160, 12 atoms per unit cell). The observed superlattice reflections correspond to the 003 reflection of this structure. The resulting structure factor for the 0003 reflection (hexagonal axes) contains only contributions of $\mathrm{Ga}$ and $\mathrm{In}$. This is true if the free parameter

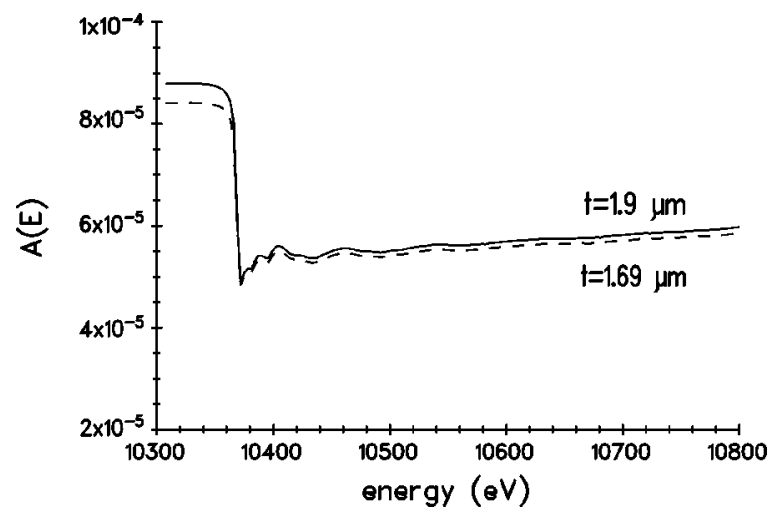

FIG. 4. Absorption correction function $A(E)$ for the superlattice reflections vs photon energy, (sample 48/2: $t=1.9 \mu \mathrm{m}$; sample 69/2: $t=1.69 \mu \mathrm{m})$.

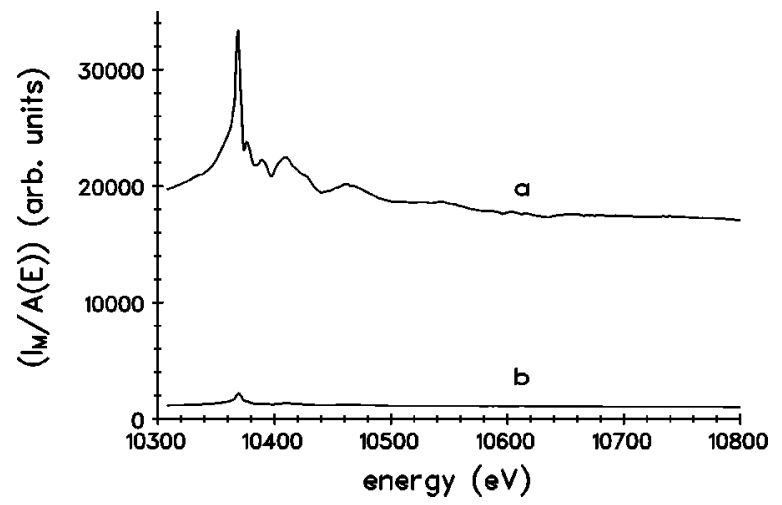

FIG. 5. Absorption-corrected DAFS reflection intensities $(R 3 m$ 003 superlattice reflection, hexagonal $c$ axis parallel to [11ㅣ (a) and $[\overline{1} 11]$ (b) directions of the sphalerite structure of specimen $48 / 2$ ) vs photon energy.

$\mathrm{Z}$ of Wyckoff position $3 a(0,0, z)$ of $\mathrm{P}$ atoms is equal to the values of 0.125 and 0.625 , respectively. Deviations from these ideal positions would yield contributions of $\mathrm{P}$ that can be excluded experimentally by the good agreement between calculated and measured (normalized) intensities. The structure factor $F_{0}$ (smooth part without contributions of fine structure) for the 003 reflection with nonresonant atomic scattering amplitudes $f_{0 G a}$ and $f_{0 I n}$ and their resonant (anomalous) parts $f_{s G a}^{\prime}+i f_{s G a}^{\prime \prime}$ and $f_{s I n}^{\prime}+i f_{s I n}^{\prime \prime}$ (resulting in corrections of the smooth curve without contributions of fine structure) is given by

$$
F_{0}=3\left(f_{0 G a}+f_{s G a}^{\prime}+i f_{s G a}^{\prime \prime}\right)-3\left(f_{0 I n}+f_{s I n}^{\prime}+i f_{s I n}^{\prime \prime}\right),
$$

where the real part $\operatorname{Re}\left(F_{0}\right)$ and the imaginary part $\operatorname{Im}\left(F_{0}\right)$ can be combined to

$$
\operatorname{Re}\left(F_{0}\right)=3\left(f_{0 G a}+f_{s G a}^{\prime}-f_{0 I n}-f_{s I n}^{\prime}\right)
$$

and

$$
\operatorname{Im}\left(F_{0}\right)=3\left(f_{s G a}^{\prime \prime}-f_{s I n}^{\prime \prime}\right) .
$$

Taking into account the oscillating fine-structure terms of the atomic scattering amplitudes $f_{o s G a}^{\prime}$ and $f_{o s G a}^{\prime \prime}$ in our case of $\mathrm{Ga}$ as a resonantly scattering atom, the complete structure factor $F$ can be written as

$$
F=F_{0}+3\left(f_{o s G a}^{\prime}+f_{o s G a}^{\prime \prime}\right) \text {. }
$$

In accordance with Sorensen et al., ${ }^{9}$ the complex-valued fine-structure function $\chi=\chi^{\prime}+i \chi^{\prime \prime}$ is connected to the oscillating terms of the atomic scattering amplitudes by $f_{o s G a}^{\prime}$ $=f_{s G a}^{\prime \prime} \chi^{\prime}$ and $f_{o s G a}^{\prime \prime}=f_{s G a}^{\prime \prime} \chi^{\prime \prime}$. Neglecting the square terms in $f_{o s G a}^{\prime}$ and $f_{o s G a}^{\prime \prime}$, the intensity becomes

$$
I \sim\left|F_{0}\right|^{2}+2 \operatorname{Re}\left(F_{0}\right) 3 f_{o s G a}^{\prime}+2 \operatorname{Im}\left(F_{0}\right) 3 f_{o s G a}^{\prime \prime},
$$

or, with the notation of the complex-valued fine-structure function mentioned above,

$$
I \sim\left|F_{0}\right|^{2}+2 \operatorname{Re}\left(F_{0}\right) 3 f_{s G a}^{\prime \prime} \chi^{\prime}+2 \operatorname{Im}\left(F_{0}\right) 3 f_{s G a}^{\prime \prime} \chi^{\prime \prime} .
$$

Since we directly follow Sorensen et al., we have to note here that a distinction is not made between contributions of all of the electrons and of the core electrons excited at the $\mathrm{Ga}$ 


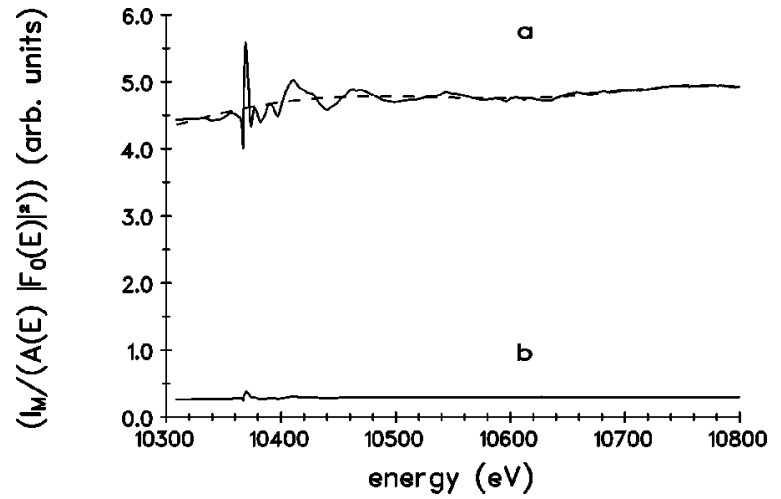

FIG. 6. DAFS reflection intensities related to the theoretical reflection intensities $\left|F_{0}\right|^{2}(R 3 m 003$ superlattice reflection, hexagonal $c$ axis parallel to [1 $\overline{1} 1]$ (a) and [ $\overline{1} 11]$ (b) directions of the sphalerite structure of specimen 48/2).

$K$ edge to $f_{s G a}^{\prime \prime}$. The $f_{s G a}^{\prime \prime}$ used here stands for the core electrons alone. In the present case this neglection can cause an error of the coordination numbers calculated from the fine structure in the order of $10 \%$. Finally for the $(\mathrm{Ga}, \mathrm{In}) \mathrm{P} 003$ reflection $(R 3 m$ symmetry) we derive

$$
\begin{aligned}
I \sim & \left|F_{0}\right|^{2}+18\left[\left(f_{0 G a}+f_{s G a}^{\prime}-f_{0 I n}-f_{s I n}^{\prime}\right) f_{o s G a}^{\prime}\right. \\
& \left.+\left(f_{s G a}^{\prime \prime}-f_{s I n}^{\prime \prime}\right) f_{o s G a}^{\prime \prime}\right]
\end{aligned}
$$

or

$$
\begin{aligned}
I \sim & \left|F_{0}\right|^{2}+18 f_{s G a}^{\prime \prime}\left[\left(f_{0 G a}+f_{s G a}^{\prime}-f_{0 I n}-f_{s I n}^{\prime}\right) \chi^{\prime}\right. \\
& \left.+\left(f_{s G a}^{\prime \prime}-f_{s I n}^{\prime \prime}\right) \chi^{\prime \prime}\right] .
\end{aligned}
$$

The resulting quotients of the absorption-corrected measured reflection intensities $I_{M}(E) / A(E)$ and $\left|F_{0}(E)\right|^{2}$ calculated with the atomic scattering factors for $\mathrm{Ga}$ and $\mathrm{In},{ }^{14}$ and the corrections calculated according to Cromer and Liberman, ${ }^{17}$ are shown in Fig. 6 for sample 48/2. Normalized absorptioncorrected measured intensities oscillating around $\left|F_{0}\right|^{2}$ of these superlattice reflection intensities are shown as example in Fig. 7. DAFS $\chi^{\prime}$ and $\chi^{\prime \prime}$ signals were derived using an iterative Kramers-Kronig algorithm and adapting the model function

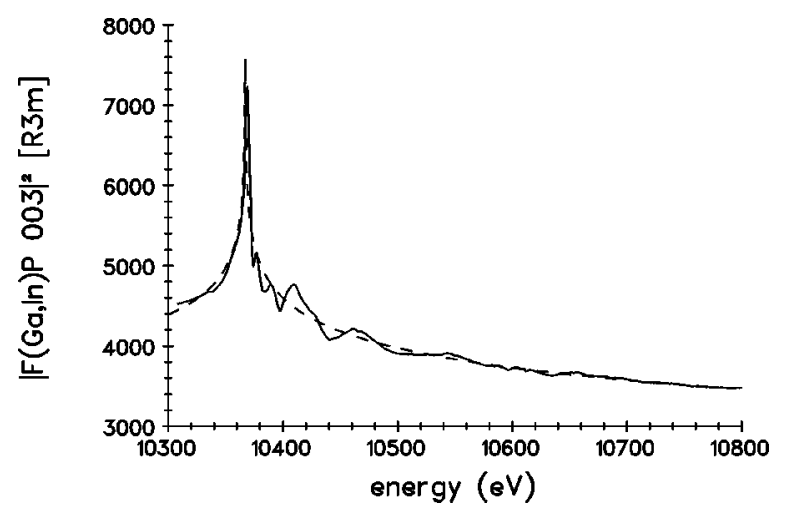

FIG. 7. Normalized intensities (full line) and $\left|F_{0}\right|^{2}$ (dashed line) for the $R 3 m 003$ superlattice reflection, hexagonal $c$ axis parallel to [111] direction of the sphalerite structure of specimen $48 / 2$.

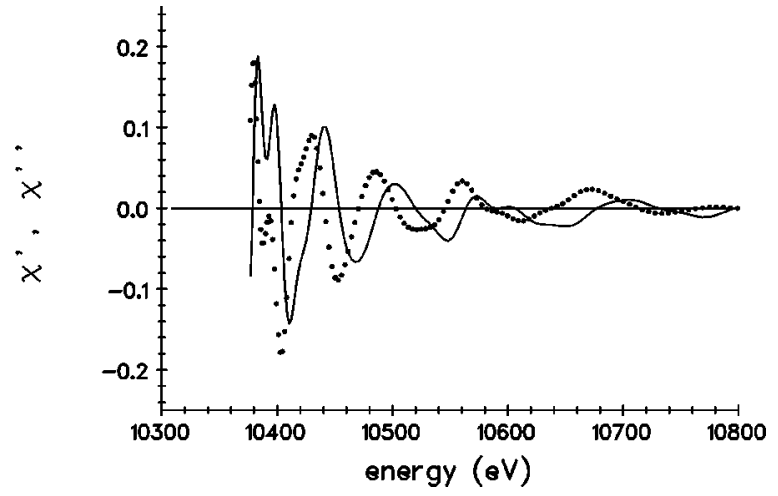

FIG. 8. Real part $\chi^{\prime}$ (line) and imaginary part $\chi^{\prime \prime}$ (dots) of the complex valued fine structure function vs photon energy ( $R 3 m 003$ superlattice reflection, hexagonal $c$ axis parallel to [111] and [111] directions of the sphalerite structure of specimen 48/2).

$$
I-\left|F_{0}\right|^{2}=A \chi^{\prime}+B \chi^{\prime \prime}
$$

to the measured extracted DAFS. The values $A$ and $B$ are equal to about 20 and 1, respectively. Therefore a first approximate value for $\chi^{\prime}$ can be obtained by dividing the measured fine-structure contribution to $I(E)$ by $A$ neglecting $\chi^{\prime \prime}$. A Kramers-Kronig transformation gives $\chi^{\prime \prime}$ from $\chi^{\prime}$. In our case one further iteration step already resulted in stable $\chi^{\prime}$ and $\chi^{\prime \prime}$ signals within the accuracy of the intensity measurement. The results from iterative Kramers-Kronig analysis are shown in Fig. 8 (filtered with respect to high-frequency noise). For the quantitative Ga $K$ XAFS and DAFS analysis we calculated the theoretical fine structure functions of the ideal structures of $(\mathrm{Ga}, \mathrm{In}) \mathrm{P}$ using the program MODEX, ${ }^{16}$ which is based on a single-scattering plane-wave model, the theoretical scattering phase shifts, and amplitudes of McKale et al. Assuming a lattice parameter $\mathrm{a}=0.566 \mathrm{~nm}$ (Ref. 15) for the ZnS-type structure $(\mathrm{Ga}, \mathrm{In}) \mathrm{P}$, interatomic distances and effective coordination numbers of the neighborhood of $\mathrm{Ga}$ atoms are compiled in Table III, which also contains the effective coordination numbers in the case of ordering considering that the electrical field vector realized in our experiment was perpendicular to the plane spanned by the $\langle 111\rangle$ and [001] vectors. The contributions expected from backscatterers to the Ga $K$ XAFS shown in Figs. 9 and 10; however, were calculated with the parameters from Table IV resulting from the fit to the measured XAFS values. From Figs. 9 and 10 we can see the following.

(1) The Ga $K$ XAFS and DAFS are dominated by the contributions of the first $\mathrm{P}$ neighbors of $\mathrm{Ga}$.

TABLE III. Local structure parameters of the Ga neighborhood in sphalerite $(\mathrm{Ga}, \mathrm{In}) \mathrm{P}$ and in case of $R 3 m$-type ordering as calculated from long-range ordered structure model.

\begin{tabular}{cccc}
\hline \hline $\begin{array}{c}\text { Neighbor } \\
\text { of Ga }\end{array}$ & Distance & $\begin{array}{c}\text { Effective } \\
\text { coordination } \\
\text { number in } F \overline{4} 3 m\end{array}$ & $\begin{array}{c}\text { Effective } \\
\text { coordination } \\
\text { number in } R 3 m\end{array}$ \\
\hline $\mathrm{P}$ & $0.245 \mathrm{~nm}$ & 4 & 4 \\
$\mathrm{Ga}$ & $0.400 \mathrm{~nm}$ & 6 & 9 \\
$\mathrm{In}$ & $0.400 \mathrm{~nm}$ & 6 & 3 \\
$\mathrm{P}$ & $0.469 \mathrm{~nm}$ & 12 & 12 \\
\hline \hline
\end{tabular}




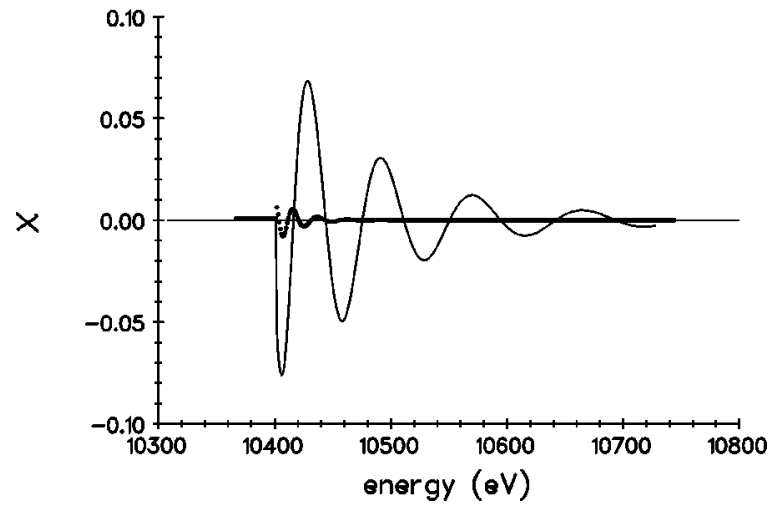

FIG. 9. Expected contributions of different neighbors to the theoretical Ga $K$ XAFS/DAFS (imaginary part): $\mathrm{P}$ neighbors at 0.24 $\mathrm{nm}$ (line) and $\mathrm{P}$ atoms at $0.47 \mathrm{~nm}$ (dots).

(2) The contributions of the next nearest $\mathrm{P}$ neighbors of $\mathrm{Ga}$ are small. They are restricted to the energy region corresponding to small values of the wave vector.

(3) The contributions of the nearest Ga and In backscatterers superimpose nearly opposite in phase for the main part of the interesting wave-vector region. This means the nearest $\mathrm{Ga}$ and In neighbors are not recognizable as "backscatterer shells," as expected from isolated estimation in the Fourier transform (Fig. 11). Figure 12, however, proves, by comparison of the sum of the Fourier transforms of Fig. 11 and the Fourier transform of the complete-fine structure function (sum of all contributions in the $E / k$ scale, respectively), that only the first $\mathrm{P}$ neighbors to form an isolated backscatterer shell. The other interference structures in the Fourier transform cannot be assigned definitely to such shells.

XAFS and DAFS analysis went on by the following steps.

(i) Fourier transform of the fine-structure function (weighted by $k^{3}$ to obtain a sufficiently high position resolution of the neighbors near $0.4 \mathrm{~nm}$ ).

(ii) Separate backtransformation of the next $\mathrm{P}$ neighbor contributions $(0.12$ to $0.25 \mathrm{~nm})$ and of the adjacent region $(0.25$ to $0.60 \mathrm{~nm})$ containing the contributions mainly of $\mathrm{Ga}$ and In backscatterers (back filtering). In addition, the backtransformation of the whole range $(0.12$ to $0.60 \mathrm{~nm})$ gives a filtered (smoothed) fine-structure function. With the computer program mentioned above the local structure models for the Ga-P coordination and for the Ga-Ga/In coordination

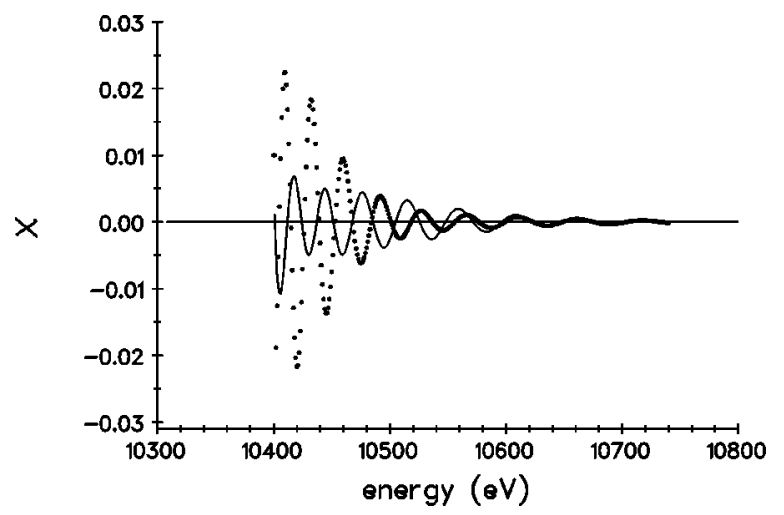

FIG. 10. Expected contributions of different neighbors to the theoretical Ga $K$ XAFS/DAFS (imaginary part): Ga atoms at 0.394 $\mathrm{nm}$ (line), and In atoms at $0.402 \mathrm{~nm}$ (dots).
TABLE IV. Local structure model of the Ga neighborhood in $(\mathrm{Ga}, \mathrm{In}) \mathrm{P}$ as derived from XAFS data.

\begin{tabular}{cccc}
\hline \hline $\begin{array}{c}\text { Neighbor } \\
\text { of Ga }\end{array}$ & $\begin{array}{c}\text { Effective } \\
\text { coordination } \\
\text { number }\end{array}$ & Distance & $\sigma^{2}$ \\
\hline $\mathrm{P}_{1}$ & $2.2 \pm 0.5$ & $0.239 \pm 0.002 \mathrm{~nm}$ & $0.00006 \mathrm{~nm}^{2}$ \\
$\mathrm{Ga}$ & $6.5 \pm 1.3$ & $0.394 \pm 0.005 \mathrm{~nm}$ & $0.00024 \mathrm{~nm}^{2}$ \\
$\mathrm{In}$ & $5.5 \pm 1.1$ & $0.402 \pm 0.005 \mathrm{~nm}$ & $0.00043 \mathrm{~nm}^{2}$ \\
\hline \hline
\end{tabular}

(paying attention to the next-nearest Ga-P coordination) were fitted separately to the back-filtered functions. Successively the partial models were summed up and fitted to the filtered fine-structure function resulting from the backtransformation of the whole range.

The comparison between filtered partial/sum shells and fitted data based on the structure models in Figs. 13 and 14 shows good agreement. Structure considerations lead to one run the fit procedure under the constraint of an effective $\mathrm{Ga} / \mathrm{In}$ coordination number 12 [at the $\mathrm{ZnS}$-type structure of $(\mathrm{Ga}, \mathrm{In}) \mathrm{P}$, one $\mathrm{Ga}$ atom has on the average six $\mathrm{Ga}$ and six In neighbors]. Table IV contains the local structure parameters for a Ga neighborhood resulting from the XAFS intensity of the whole $(\mathrm{Ga}, \mathrm{In}) \mathrm{P}$ layer. The DAFS results (example of Fourier transform in Fig. 15 gained from superlattice reflection of the specimen 48/2 in the way described for XAFS) in comparison to XAFS show significant differences relating to the contributions of the next $P$ neighbors. Fitting of the structure model of the ordered domains to experiment was performed, again constraining the effective $\mathrm{Ga} / \mathrm{In}$ coordination number 12. However, since Ga and In ordering occurs on separate planes, there will be weighted contributions of nine $\mathrm{Ga}$ atoms and three In atoms as next-nearest neighbors in $\langle 111\rangle$ directions of the $\mathrm{ZnS}$-type structure of $(\mathrm{Ga}, \mathrm{In}) \mathrm{P}$ following one another. This relation between the $\mathrm{Ga}$ and In effective coordination numbers should occur only with large domains combined with the ideal $R 3 \mathrm{~m}$ structure (ordering) and a complete polarization of the synchrotron radiation perpendicular to the $R 3 m$ hexagonal $c$ axis [parallel to $\langle 111\rangle$ directions of $F \overline{4} 3 m(\mathrm{Ga}, \mathrm{In}) \mathrm{P}]$. These conditions are not exactly fulfilled, but we can discuss trends looking at deviations from the ideal (expected) effective coordination num-

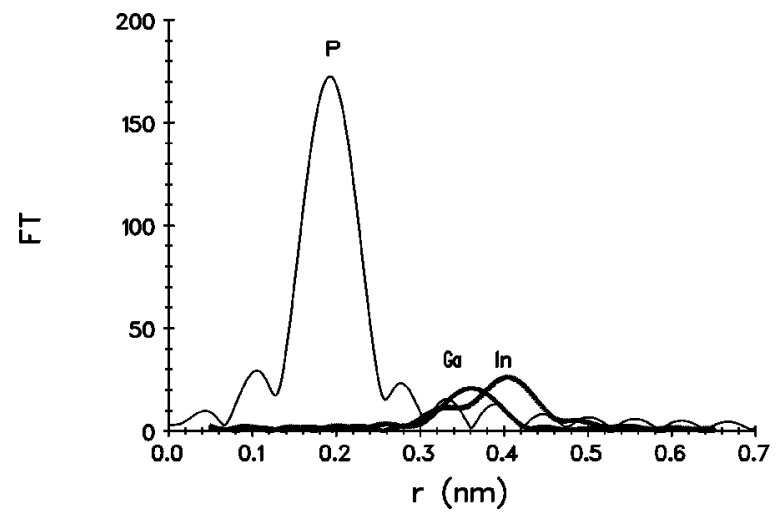

FIG. 11. Fourier transforms FT( $r)$ of the partial contributions of P, Ga and In in Figs. 9 and 10 to the Ga $K$ XAFS/DAFS. $r$ is the interatomic distance with $\mathrm{Ga}$ in the center. 


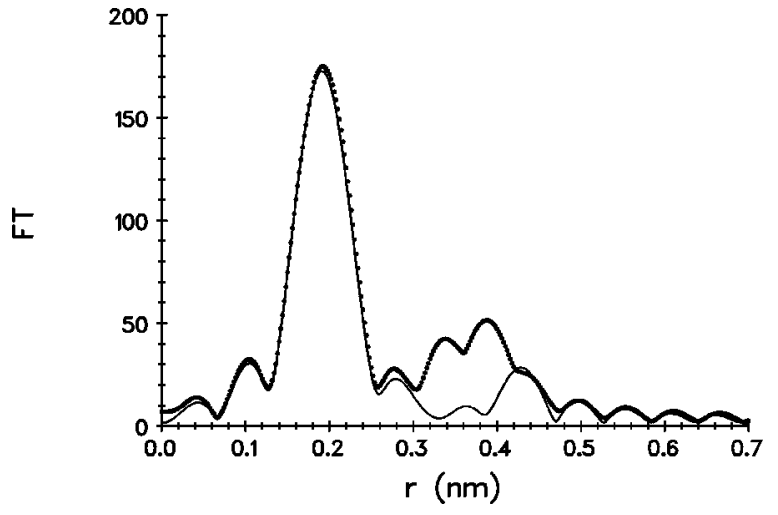

FIG. 12. Sum of Fourier transforms FT( $r$ ) of Fig. 11 (dots) and of the total XAFS signal (line).

bers compared to XAFS results for the whole layer that correspond to the expected relation within the error of measurement, and may also analyze data which are strongly influenced by the theoretical scattering cross-section data. ${ }^{18}$ The parameters of the structure models which also prove the Ga $K$ fine-structure functions of the DAFS experiments at superlattice reflections are listed in Table V.

\section{DISCUSSION}

Two standard models of local structure in solid solution crystals are of relevance here: The virtual-crystal model (VCM) describes the alloy as an arrangement of atoms at ideal sites in the corresponding unit cell. The assumption is that atoms remain at their sites despite compression or expansion of the unit cell caused by alteration of the composition. Various authors (Refs. 9 and 19) gave examples of nonconformity of the VCM with the results of XAFS experiments obtained with alloy semiconductors which had overcome the averaging character of diffraction experiments. The latter, however, could be better understood by applying the local structure model (LSM) that describes local distortions of the structure, and takes into consideration that the binary components with tetrahedral bonds tend to compensate for changes of bonding angles by maintaining the bond lengths. Sorensen et al. ${ }^{9}$ showed that their results of DAFS experiments of $\operatorname{In}_{x} \mathrm{Ga}_{1-x}$ As were comprehensible only on

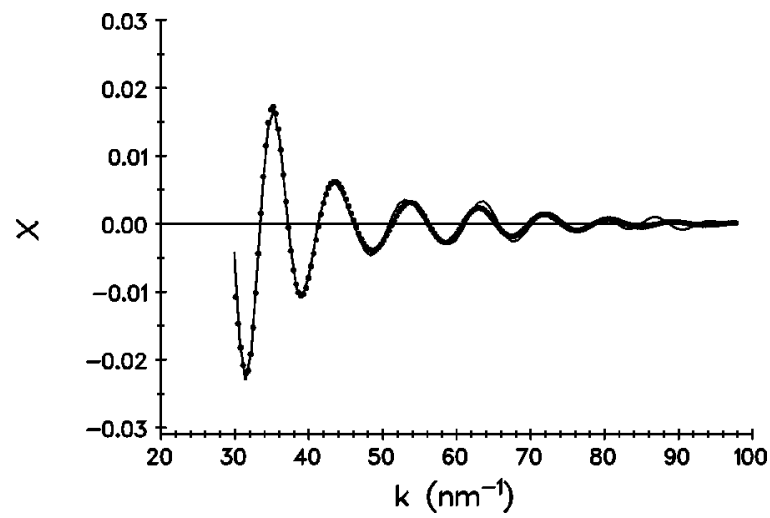

FIG. 13. Filtered partial/sum shell data (dots) and fitted data basing on the structure model (line) (Ga $K$ XAFS of specimen 48/2) excluding nearest $\mathrm{P}$ neighbors of $\mathrm{Ga}$.

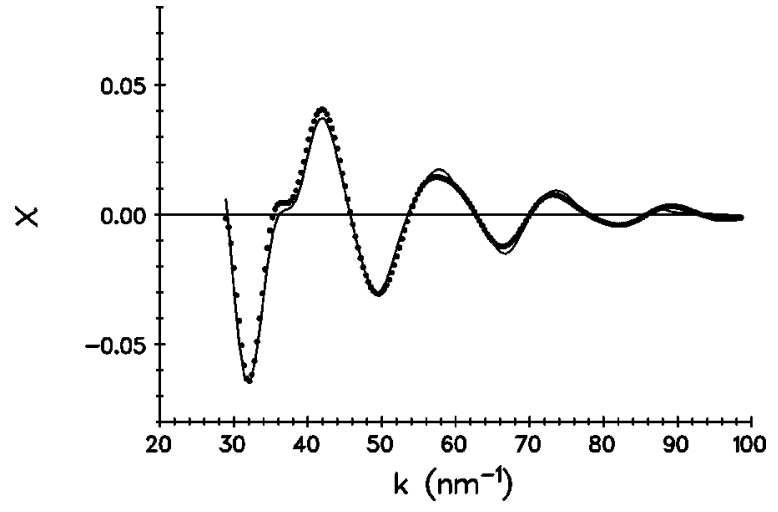

FIG. 14. Filtered partial/sum shell data (dots) and fitted data basing on the structure model (line) (Ga $K$ XAFS of specimen 48/ 2). Parameters of the structure model are listed in Table III.

the basis of the LSM. In the present case the (Ga,In)P solid solution can be understood as a composition of Ga-P and In-P components. In case of $\mathrm{GaP}$ the nearest-neighbor distance Ga-P amounts to $0.236 \mathrm{~nm}\left[\mathrm{a}_{G a P}=0.5451 \mathrm{~nm}\right.$ (Ref. 15) that should match the results of XAFS experiments for (Ga,In)P when we follow the LSM. The VCM would be appropriate to describe XAFS results which show nearest neighbor Ga-P distances of $0.245 \mathrm{~nm}$ corresponding to a lattice parameter $\mathrm{a}_{(G a, I n) P}=0.5660 \mathrm{~nm}^{15}$

Changes of bond angles cause changes of the angle between bond direction and the direction of the polarization vector of the synchrotron radiation. This may influence the effective coordination numbers of XAFS and, therefore, DAFS results.

The average nearest-neighbor distance Ga-P for the whole (Ga,In)P layer of about $0.239 \mathrm{~nm}$ (as a result of XAFS) is in between the two situations discussed above. Obviously, within the experimental error our value agrees better with the LSM than with the VCM. Any superposition of contributions has to take into account the weight which is due to the orientation of the electric-field vector. Thus the small value of 2.2 nearest $\mathrm{P}$ neighbors of $\mathrm{Ga}$ (4.0 were expected) could be understood by changes of bond directions. However, experimental effects due to the limited takeoff angle of the fluorescence cannot be excluded. If the signal were in fact coming

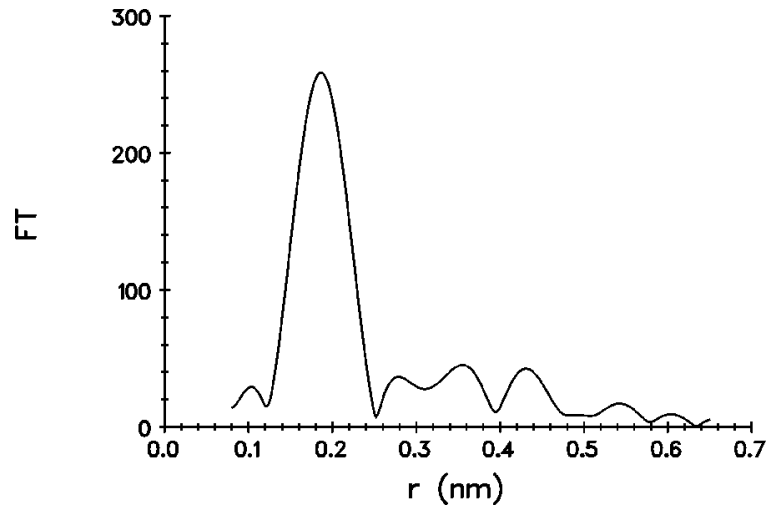

FIG. 15. Fourier transform FT( $r)$ of Ga $K$ DAFS: hexagonal $c$ axis of the $R 3 \mathrm{~m}$ structure parallel to the [111] direction of the sphalerite structure of specimen $48 / 2$. 
TABLE V. Local structure models for ordered regions as derived from DAFS data. (a) Specimen 60/2, $R 3 m c$ axis (hex. axis) parallel to the $F \overline{4} 3 m$ [11̄1] direction. (b) Specimen 69/2, $R 3 m c$ axis (hex. axis) parallel to the $F \overline{4} 3 m$ [1 $\overline{1} 1]$ direction. (c) Specimen $48 / 2 R 3 m c$ axis (hex. axis) parallel to $F \overline{4} 3 m$ [1 $1 \overline{1} 1]$ direction. (d) Specimen 48/2, $R 3 m c$ axis (hex. axis) parallel to the $F \overline{4} 3 m$ [ $\overline{1} 11]$ direction.

\begin{tabular}{|c|c|c|c|}
\hline $\begin{array}{c}\text { Neighbor } \\
\text { of } \mathrm{Ga}\end{array}$ & $\begin{array}{c}\text { Effective } \\
\text { coordination } \\
\text { number }\end{array}$ & Distance & $\sigma^{2}$ \\
\hline \multicolumn{4}{|c|}{ (a) } \\
\hline $\mathrm{P}_{1}$ & $4.1 \pm 0.9$ & $0.246 \pm 0.002 \mathrm{~nm}$ & $0.00007 \mathrm{~nm}^{2}$ \\
\hline $\mathrm{Ga}$ & $6.5 \pm 1.3$ & $0.395 \pm 0.005 \mathrm{~nm}$ & $0.00016 \mathrm{~nm}^{2}$ \\
\hline In & $5.5 \pm 1.1$ & $0.411 \pm 0.005 \mathrm{~nm}$ & $0.00015 \mathrm{~nm}^{2}$ \\
\hline \multicolumn{4}{|c|}{ (b) } \\
\hline $\mathrm{P}_{1}$ & $4.0 \pm 0.8$ & $0.246 \pm 0.002 \mathrm{~nm}$ & $0.00007 \mathrm{~nm}^{2}$ \\
\hline $\mathrm{Ga}$ & $7.0 \pm 1.4$ & $0.396 \pm 0.005 \mathrm{~nm}$ & $0.00015 \mathrm{~nm}^{2}$ \\
\hline In & $5.0 \pm 1.0$ & $0.412 \pm 0.005 \mathrm{~nm}$ & $0.00018 \mathrm{~nm}^{2}$ \\
\hline \multicolumn{4}{|c|}{ (c) } \\
\hline $\mathrm{P}_{1}$ & $4.2 \pm 0.9$ & $0.245 \pm 0.002 \mathrm{~nm}$ & $0.00006 \mathrm{~nm}^{2}$ \\
\hline $\mathrm{Ga}$ & $7.1 \pm 1.5$ & $0.398 \pm 0.005 \mathrm{~nm}$ & $0.00014 \mathrm{~nm}^{2}$ \\
\hline In & $4.9 \pm 1.0$ & $0.412 \pm 0.005 \mathrm{~nm}$ & $0.00015 \mathrm{~nm}^{2}$ \\
\hline \multicolumn{4}{|c|}{ (d) } \\
\hline $\mathrm{P}_{1}$ & $4.2 \pm 0.9$ & $0.246 \pm 0.002 \mathrm{~nm}$ & $0.00005 \mathrm{~nm}^{2}$ \\
\hline $\mathrm{Ga}$ & $7.9 \pm 1.6$ & $0.399 \pm 0.005 \mathrm{~nm}$ & $0.00016 \mathrm{~nm}^{2}$ \\
\hline In & $4.1 \pm 0.9$ & $0.413 \pm 0.005 \mathrm{~nm}$ & $0.00014 \mathrm{~nm}^{2}$ \\
\hline
\end{tabular}

from the entire depth of the film and somewhat from the substrate, then additional self-absorption not considered here would have a significant damping effect on the fluorescence XAFS. Another possible explanation for the deviation from the expected value is the damping of the XAFS amplitude caused by static and thermal disorder. Deviations from symmetric pair distribution functions caused by local distortions may yield comparable effects. In the present case the dependence of the backscattering amplitude of $\mathrm{P}$ on wave vector $\mathbf{k}$ at lower $\mathbf{k}$ values is similar to the influence of damping on a constant backscattering amplitude, because it is difficult to distinguish between influences of coordination number and damping. Nevertheless the deviation from the expected value is an open question which should be the subject of further investigation. Thus the fit result may be formulated more generally by stating that the nearest neighbors of Ga deviate from ideal positions in the unit cell throughout the whole (Ga,In)P layer, while showing nearest-neighbor distances similar to those of the binary compound GaP.

DAFS experiments with different samples (comparable orientation of the polarization direction of the electric-field vector as discussed in case of XAFS experiments) yielded nearest-neighbor distances between 0.245 and $0.246 \mathrm{~nm}$. These values match the VCM. The effective coordination numbers of about four next-nearest $\mathrm{P}$ neighbors of $\mathrm{Ga}$ agree with the values expected for an occupation of ideal positions of the compound. The nearest neighborhood of $\mathrm{Ga}$ in the whole (Ga,In)P layer can be described by the LSM, while in the ordered regions Ga is surrounded according to the VCM.

Nearest Ga-Ga neighbor distances (0.394-0.399 nm) resulting from XAFS and DAFS measurements are comparable within the error limits, and are less than the average value expected for $(\mathrm{Ga}, \mathrm{In}) \mathrm{P}(0.400 \mathrm{~nm})$. The nearest Ga-In neighbor distance for the whole (Ga,In)P layer (result of XAFS) amounts to $0.402 \mathrm{~nm}$, which is comparable to the average value expected. For the ordered regions from DAFS nearest Ga-In neighbor distances of $0.411-0.413 \mathrm{~nm}$ have been derived that are significantly larger than the average value of (Ga,In)P. One has to consider that in ordered regions the nearest In neighbors of Ga form separate planes, and only neighbors in particular directions contribute to the DAFS. All this indicates a distortion parallel to the $c$ axis of the ordered $R 3 m$ phase (hexagonal setting). This agrees well with the observed Bragg angle shift of all superlattice reflections involved compared to random packing.

From superlattice reflection intensities as well as from the ratio of effective $\mathrm{Ga} / \mathrm{In}$ coordination numbers (next-nearest Ga neighbors), we conclude that coherently ordered regions possibly enlarge with increasing growth temperature. This connection found for three samples seems to be a hint for further investigation. We observed a change from 6.5:5.5 (Ga:In) in the case of specimen 60/2 (comparable to the average result of XAFS) to 7.0:5.0 in the case of specimen $69 / 2$ and 7.1:4.9 as well as 7.9:4.1 in the case of specimen 48/2. There is no significant difference in ordering for both $\langle 111\rangle$ directions, while differences of superlattice reflection intensities usually hint at different numbers of coherently scattering atoms.

\section{CONCLUSIONS}

The average coordination of $\mathrm{Ga}$ in partially ordered epitaxial $(\mathrm{Ga}, \mathrm{In}) \mathrm{P}$ layers has been determined by XAFS experiments, while the local structure around $\mathrm{Ga}$ in particularly ordered regions (a few percent of the whole volume) was explored by DAFS experiments using superlattice reflections of these ordered regions. The local structure around $\mathrm{Ga}$ in the whole $(\mathrm{Ga}$,In)P layer $(F \overline{4} 3 m)$ can be described by the local structure model. Contrary to that, results in the nearest neighborhood of $\mathrm{Ga}$ in the ordered regions $(R 3 \mathrm{~m})$ follow the virtual-crystal model. This means that ordering not only causes a reduction of symmetry but causes changes in bonding characteristics. Thus the solid solution in the ordered region can no longer be considered as a simple superposition of the binary components $\mathrm{GaP}$ and InP. Hence ordering of (Ga,In)P may be driven by electronic characteristics of interfaces between ordered and disordered structures resulting from lattice expansion in the directions of ordering.

\section{ACKNOWLEDGMENTS}

Our thanks are due to Dr. V. Gottschalch (Institute of Inorganic Chemistry, Leipzig University) for providing the (Ga,In)P samples. The authors are also indebted to J. Weigelt and R. Frahm for inspiring discussions and their assistance with the measurements. Financial support by the BMBF is gratefully acknowledged. 
${ }^{1}$ R. H. Horng and M. K. Lee, J. Appl. Phys. 71, 1513 (1992).

${ }^{2}$ O. Ueda, M. Takikawa, J. Komeno, and I. Umebu, Jpn. J. Appl. Phys., Part 2 26, L1824 (1987).

${ }^{3}$ O. Ueda, M. Takechi, and J. Komeno, Appl. Phys. Lett. 54, 2312 (1989).

${ }^{4}$ E. Morita, M. Ikeda, O. Kumagai, and K. Kaneko, Appl. Phys. Lett. 53, 2164 (1988).

${ }^{5}$ D. C. Meyer, J. Weigelt, R. Frahm, K. Richter, and P. Paufler (unpublished).

${ }^{6}$ D. C. Meyer, A. Seidel, K. Richter, J. Weigelt, R. Frahm, and P. Paufler (unpublished).

${ }^{7}$ I. Arcon, A. Kodre, D. Glavic, and M. Hribar, J. Phys. (Paris), Colloq. 48, C9-1105 (1987).

${ }^{8}$ H. Stragier, J. O. Cross, J. J. Rehr, L. B. Sorensen, C. E. Bouldin, and J. C. Woicik, Phys. Rev. Lett. 69, 3064 (1992).

${ }^{9}$ L. B. Sorensen, J. O. Cross, M. Newville, B. Ravel, J. J. Rehr, H. Stragier, C. E. Bouldin, and C. E. Woicik, in Resonant Anomalous X-Ray Scattering, edited by G. Materlik, C. J. Sparks, and K. Fischer (Elsevier, Amsterdam, 1994), pp. 389-420.
${ }^{10}$ M. G. Proietti, H. Renevier, J. F. Berar, V. Dalakas, J. L. Hodeau, G. Armelles, and J. Garcia, J. Phys. (Paris), Colloq. 7, C2-749 (1997).

${ }^{11}$ D. C. Meyer, K. Richter, A. Seidel, J. Weigelt, R. Frahm, and P. Paufler, J. Synchrotron Radiat. 5, 1275 (1998).

${ }^{12}$ V. Gottschalch, R. Franzheld, I. Pietzonka, R. Schwabe, G. Benndorf, and G. Wagner, Cryst. Res. Technol. 32, 69 (1997).

${ }^{13}$ D. C. Meyer, K. Richter, A. Seidel, K.-D. Schulze, R. Sprungk, and P. Paufler (unpublished).

${ }^{14}$ International Tables for X-Ray Crystallography (Reidel, Dordrecht, 1985), Vol. III.

${ }^{15}$ S. Adachi, J. Appl. Phys. 53, 8775 (1982).

${ }^{16}$ S. Moldenhauer, Diploma thesis, TU Dresden, 1992.

${ }^{17}$ D. T. Cromer and D. Liberman, J. Chem. Phys. 53, 1891 (1970).

${ }^{18}$ A. G. McKale, B. W. Veal, A. P. Paulikas, S.-K. Chan, and G. S. Knapp, J. Am. Chem. Soc. 208, 3763 (1988).

${ }^{19}$ Y. Takeda, H. Oyanagi, and A. Sasaki, J. Appl. Phys. 68, 4513 (1990). 